\title{
Time-Resolved Studies of Laser Damage Processes in DKDP Crystals
}

H. Jiang, J. McNary, H.W.K. Tom, M. Yan, H. Radousky and S. Demos

This article was submitted to

Laser-Induced Damage in Optical Materials 1999

Boulder, CO

October 4-7, 2000

U.S. Department of Energy

February 12, 2000

Lawrence

Livermore

National

Laboratory 


\section{DISCLAIMER}

This document was prepared as an account of work sponsored by an agency of the United States Government. Neither the United States Government nor the University of California nor any of their employees, makes any warranty, express or implied, or assumes any legal liability or responsibility for the accuracy, completeness, or usefulness of any information, apparatus, product, or process disclosed, or represents that its use would not infringe privately owned rights. Reference herein to any specific commercial product, process, or service by trade name, trademark, manufacturer, or otherwise, does not necessarily constitute or imply its endorsement, recommendation, or favoring by the United States Government or the University of California. The views and opinions of authors expressed herein do not necessarily state or reflect those of the United States Government or the University of California, and shall not be used for advertising or product endorsement purposes.

This is a preprint of a paper intended for publication in a journal or proceedings. Since changes may be made before publication, this preprint is made available with the understanding that it will not be cited or reproduced without the permission of the author.

This report has been reproduced directly from the best available copy.

Available to DOE and DOE contractors from the

Office of Scientific and Technical Information

P.O. Box 62, Oak Ridge, TN 37831

Prices available from (423) 576-8401

http:/ / apollo.osti.gov/bridge/

Available to the public from the National Technical Information Service

U.S. Department of Commerce 5285 Port Royal Rd., Springfield, VA 22161 http://www.ntis.gov/

OR

Lawrence Livermore National Laboratory Technical Information Department's Digital Library http://www.llnl.gov/tid/Library.html 
UCRL-JC-137641

\title{
Time-resolved studies of laser damage processes in DKDP crystals
}

\author{
Hongbing Jiang, Jason McNary, and Harry W.K. Tom, \\ Dept. of Physics, UC Riverside, Riverside, CA \\ and \\ Ming Yan, Harry Radousky, and Stavros Demos, \\ Lawrence Livermore National Laboratory, Livermore, CA
}

\begin{abstract}
We have used a 3-color imaging technique to obtain time-resolved series of images during nanosecond laser damage in bulk DKDP crystals. In contrast to single-pump, single-probe time-resolved imaging techniques, we are able to correlate behavior during single damage events. This enables us to observe a range of morphological dynamics that is otherwise difficult to study, including: the propagation of elastic sound waves and the liquid/solid melt front from the damage nucleation site and the dynamics of crack formation and propagation.
\end{abstract}

One of the experimental difficulties in studying the dynamics of laser-induced damage is that breakdown occurs by a stochastic process at a variety of defect sites and therefore there can be an order of magnitude difference in the amount of energy deposited at a damage nucleation site even for wellcharacterized laser pulses. This pulse-to-pulse variability makes it very difficult to use ultrafast pumpprobe techniques to time-resolve the damage dynamics. We have used a 3-color imaging technique to obtain a series of 3 time-resolved images during damage induced by a single pump pulse. This allows us to follow morphological dynamics on an ultrafast timescale for individual events for the first time. We have used this technique to study nanosecond laser-induced damage in the bulk of DKDP (deuterated KDP or KD*P). We have obtained series of images during the first $80 \mathrm{~ns}$ after the damage pulse and observed the propagation of sound waves, the dynamics of the liquid/solid melt front, and crack formation and propagation. We find that irreversible morphological damage (cracks) are due to the rapid expansion of the liquid/solid melt front and that crack propagation essentially stops when the melt front stops expanding. While morphological changes do occur during the cooling phase, most of the optically significant damage is already completed during the first $50 \mathrm{~ns}$ after the damage pulse.

The DKDP samples were grown at the Lawrence Livermore National Laboratory (LLNL) by the rapid-growth technique.[1] They were cut to $1 \mathrm{X} 1 \mathrm{X} 1 \mathrm{~cm}^{3}$ and optically polished on all 6 sides at LLNL. The surfaces were also diamond-turned to raise the surface damage threshold. The laser pump and probe pulses were derived from a Q-switched Nd:YAG laser operating in a seeded single longitudinal mode with a $15 \mathrm{~ns}$ FWHM smooth temporal waveform. The imaging setup is shown in Fig. 1. The pump (damage pulse) was obtained using the fundamental wavelength at $1.06 \mu \mathrm{m}$. We focussed the pump pulse into the bulk of the DKDP crystal to a $29 \mu \mathrm{m}$ Gaussian waist. The laser fluence was $68-80$ $\mathrm{J} / \mathrm{cm}^{2}$ and set to damage on $20-60 \%$ of the laser shots. The extremely high damage threshold observed is consistent with other recent measurements on this very high quality material. 


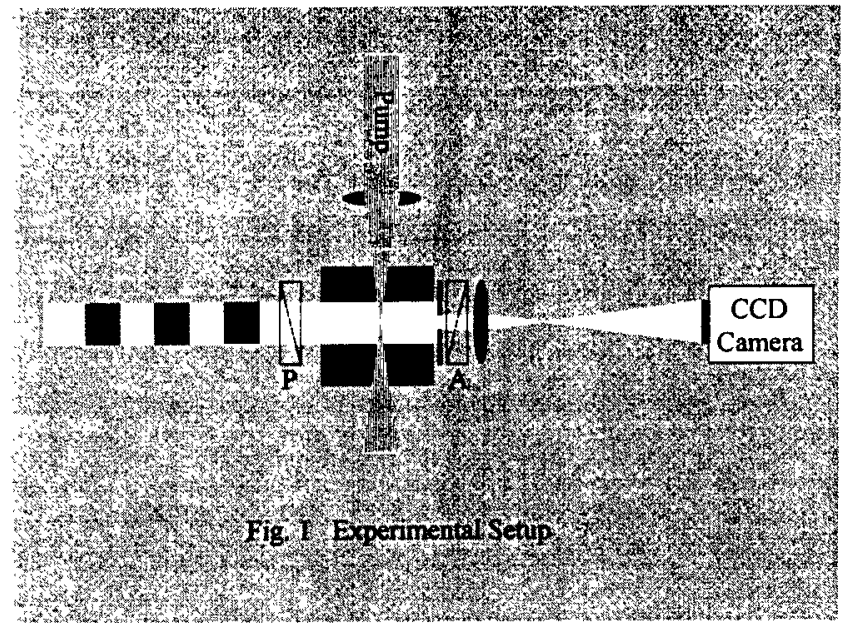

To obtain 3 time-resolved images for each pump pulse we exploited the 3 colors of an RGB CCD camera. We obtained pulses at 630 $\mathrm{nm}, 532 \mathrm{~nm}$, and $461 \mathrm{~nm}$ (corresponding to Red, Green, and Blue) by passing the focussed ND:YAG laser second-harmonic beam at 532 $\mathrm{nm}$ through a $1 \mathrm{~m}$ long cell containing methane to generate stimulated Raman scattered (SRS) light at $630 \mathrm{~nm}$ (Stokes) and $461 \mathrm{~nm}$ (AntiStokes). The $630 \mathrm{~nm}, 532 \mathrm{~nm}$, and $461 \mathrm{~nm}$ pulses were dispersed with a prism, individually time-delayed by propagation in air and then recombined to spatially overlap. All beams were spatially filtered by passing through pinholes in order to have uniform spatial profiles. The probe beams were collimated and expanded so the intensity across the $\sim 500 \mu \mathrm{m}$ image was nearly flat. The images were obtained at 90 degrees to the pump propagation direction and in transmission into a 20X microscope objective and color CCD (Panasonic GP-US502). The RGB images were separated in software. The microscope objective imaged the plane containing the center of the pump pulse beam onto the CCD. In the images that appear below, the pump (damage) pulse enters from the left of the image going to the right. Each pixel corresponds to $1.22 \times 1.22 \mu \mathrm{m}^{2}$ in the focal plane. An aperture was placed close to the exit face of the KDP sample. This restricted the $\mathrm{fH}$ to 10 . Contrast in all images may be obtained by lensing, scatter, and absorption. In addition, in some geometries the strain-induced birefringence could be imaged using crossed polarizers. The polarizers $\mathrm{P}$ and $\mathrm{A}$ (analyzer) are shown in Fig. 1. In Figs. 2 and 3 we used crossed-polarized geometries.

In Fig. 2, we show 4 images obtained (respectively from left to right) $20 \mathrm{~ns}, 40 \mathrm{~ns}, 60 \mathrm{~ns}$, and $2 \mathrm{~s}$ after the arrival of the peak of the $15 \mathrm{~ns}$ duration damage pulse. The three nanosecond time-scale images were obtained with the $\mathrm{R}, \mathrm{G}$, and $\mathrm{B}$ probe pulses, respectively, and the $2 \mathrm{~s}$ delayed image was obtained using the blue $(461 \mathrm{~nm})$ image. It is clear that much of the morphological dynamics occurs during the first $60 \mathrm{~ns}$. These images were taken using crossed polarizers. The vertical and horizontal directions in the image are along the crystalline $x=(100)$ and $y=(010)$ axes of the uniaxial crystal and the probe was propagated along the unique (optical) $\mathrm{z}$-axis (001). The polarizers were also oriented along $\mathrm{x}$ and $y$ directions and the image contrast is due to shear-induced birefringence. The coefficients of the stress-induced birefringence are well known.[2]

There are 4 main features in the morphological dynamics. First there are two expanding elliptically shaped ring patterns (vertical and horizontal) that propagate outward. This is due to the initial elastic response of the medium following the thermal expansion of the central region during the laser heating pulse. In the 40 and 60 ns image this ring is well defined and it appears like the outgoing wave when one drops a rock into a lake. By measuring the anisotropic propagation speeds in a number of images using different delay times, differing amounts of energy absorbed at the damage site, and different crystalline orientations, we confirmed that this is due to the elastic sound wave and the speed is independent of the initial energy absorbed. It is important to note that the image intensity is not linearly proportional to the path-integrated strain because of the details and symmetry of the strain-induced birefringence tensor. For example, even though there is considerable compressive strain along the $\mathrm{x}$ and $y$ axes, the birefringence is zero by symmetry so the image appears uniformly dark along these symmetry axes. 

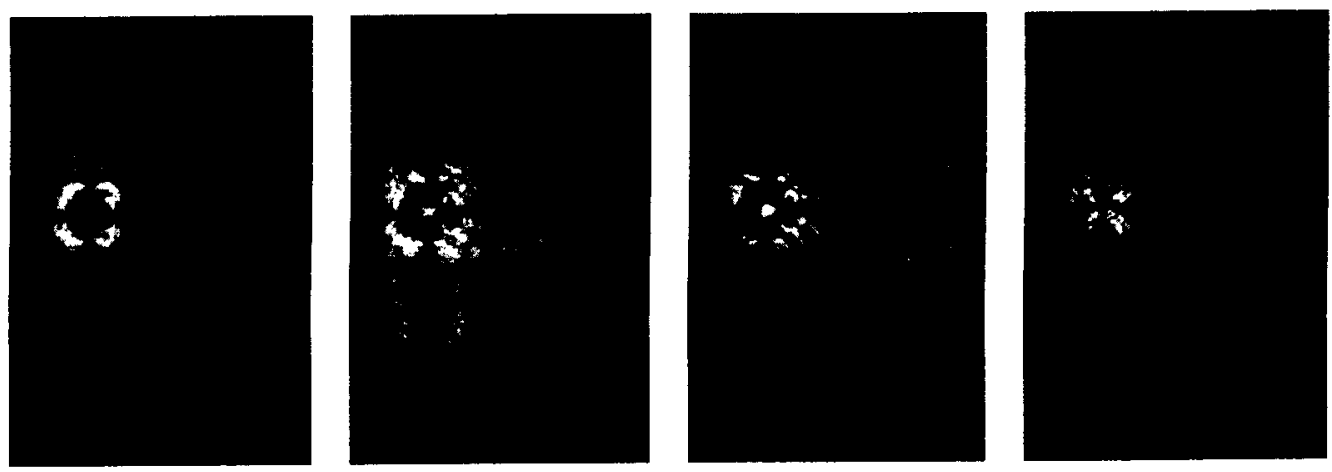

Fig. 2. Time-resolved images of laser-induced damage for a single event. From left to right, the images were taken at $20 \mathrm{~ns}, 40 \mathrm{~ns}, 60 \mathrm{~ns}$, and $2 \mathrm{~s}$ time delay after the arrival of the pump pulse. Images were obtained through crossed polarizers and are $355 \mu \mathrm{m}$ in horizontal dimension.

There is a small bright feature in the center of the damage nucleation site especially in the blue image (60 ns). This bright spot is an artifact in the probe image due to fluorescence during damage. The fluorescence is brighter when more energy is absorbed and is peaked in the blue. Because the fluorescence is emitted in all directions, it is reduced when the aperture is closed down while the rest of the image remains at constant intensity. Ignoring this fluorescence the second important feature is the dark spherical shape at the center of the damage site. This dark region is liquid. The contrast is due to either absorption or scatter since strain both in front or behind this region should make the image bright. The liquid expands much slower than the speed of sound and it appears to have stopped expanding in this damage event between 20 and 40 ns because there is no noticeable change between the size at 40 and $60 \mathrm{~ns}$. When there is more energy absorbed at the damage nucleation site, as in Fig. 3, the liquid phase expands faster and continues to expand for a longer time.

The third feature is the strain-wave induced by the expanding liquid. The liquid/solid volume ratio has not been measured but several high pressure and high temperature phases have been observed and the material has reasonably well-behaved and typical compressibility and thermal expansion coefficients.[3] If we assume the liquid/solid volume ratio is $5 \%$ and that the liquid compressibility is the same as the bulk, then the liquid must be formed at $2 \mathrm{GPa}$ higher pressure than the solid to remain at constant volume. Because the melt-front is a phase-front it is abrupt and pressure gradients of the order of several $\mathrm{GPa} / \mu \mathrm{m}$ are expected. This launches an elastic wave which propagates at the speed of sound. The region between the expanding liquid and the initial thermally-induced sound wave is "bright" in the image because new elastic strain is continuously generated at the melt front. In Fig. 3, the liquid seems to still be expanding even at 40 ns delay because the region between the initial sound wave and the melt front is strained. At 60 ns however, the melt front is no longer expanding and generating elastic waves.

The fourth feature is the formation of cracks at the liquid/solid interface. By taking images in different crystal orientations (not shown here), we have determined that there are two types of predominant cracks. One type is the largest and longest and it tends to form along the 8 equivalent $(2,2,3)$ axes. This must correspond to an "easy" plane for tearing. Velocities in the range of 1.4 to 2.1 $\mathrm{km} / \mathrm{s}$ have been measured. In addition there are many additional cracks formed nearly along the 8 equivalent $(1,1, \beta)$ directions where $\beta=0.13$ to 0.3 . It is interesting to note that most of the cracks form along $(1,1, \gamma)$ directions and not along $(1,0, \gamma)$, where $\gamma$ has some numerical value. The $(110)$ direction 



Fig. 3. Time-resolved images during laser-induced damage with higher absorbed laser fluence than in the event shown in Fig. 2. From left to right the images are taken at $20 \mathrm{~ns}, 40 \mathrm{~ns}, 60 \mathrm{~ns}$, and $2 \mathrm{~s}$ time delay. Images taken through crossed-polarizers and $355 \mu \mathrm{m}$ in horizontal dimension.

has the slowest speed of sound (and therefore pressure gradients are not relieved easily by elastic wave propagation) and maximum shear (by symmetry). Thus the maximum forces for cracking may be applied along these axes. However, there must also be some specific structural property which allows these directions to be more susceptible to fracture than others. The pressure induced by the expanding liquid is more easily relieved by tearing the solid phase to expand the liquid and compressing the surrounding solid than expanding and straining the surrounding solid uniformly in the radial direction.

There are also what appears to be a large number of very fine shorter "hair-like" cracks around the liquid and these seem relatively isotropic in distribution. They are more clearly defined in Fig. 3 (higher absorbed energy) than in Fig. 2 . These fine cracks can become fairly long but are never as long or thick as the other cracks. It would appear that once the "easy" fractures have been made, then the remaining pressure can only be relieved by a large number of relatively small cracks which have a higher energetic cost that is relatively independent of orientation.

The image taken at $2 \mathrm{~s}$ delay is dominated by the strain of plastic deformation. Comparing the volume and intensity of the image at $60 \mathrm{~ns}$ and $2 \mathrm{~s}$ in both Figs. 2 and 3, we see that the plastic deformation occurs only during the expansion of the liquid and probably only during the initial stages of expansion. It is not clear whether the plastic deformation is due only to the response of the solid to cracking or if the melt front generates an additional plastic deformation wave when the pressure gradient exceeds the elastic limit.

Upon cooling it appears the cracks formed during the expansion of the liquid do not grow but do not significantly contract either. The strain in the lattice surrounding the cracks is only slightly reduced between $50 \mathrm{~ns}$ and $2 \mathrm{~s}$. Thus the strain in the lattice holds the cracks "open" even though the interior volume that was once liquid has collapsed.

We have observed the ultrafast time-scale morphological dynamics of laser-induced damage in the bulk of DKDP by a novel time-resolved multi-probe imaging technique. The propagation speeds of phase fronts and cracks were measured and other dynamical phenomena were deduced.

In closing we would like to point out that this technique is generally applicable to the study of laserinduced processes on ultrafast time-scales down to femtoseconds with the use of femtosecond laser pulses. The multi-probe nature of this technique is especially useful for the study of material systems 
which are not easily signal averaged, not only damage of condensed media but also moving objects, rare samples, and living cells. If extended to use with 2 cameras in different orientations, one may obtain 3-d imaging capability. This technique may provide unique opportunities for imaging the dynamical response of media to 3-dimensional forces, including large shear and anisotropic forces.

This work was performed under the auspices of the U.S. Department of Energy by University of California Lawrence Livermore National Laboratory, through the Institute for Laser Science and Applications, under contract No. W-7405-Eng-48.

\section{References}

1. N. Zaitseva, J. Atherton, R. Rozsa, L. Carman, I. Smolsky, M. Runkel, R. Ryon, and L. James, "Design and benefits of continuous filtration in rapid growth of large KDP and DKDP crystals,"J. of Crystal Growth 197, 911 (1999).

2. J.J. DeYoreo and B.W. Woods, "A study of residual stress and the stress-optic effect in mixed crystals of $\mathrm{K}\left(\mathrm{D}_{\mathrm{X}} \mathrm{H}_{1-\mathrm{X}}\right)_{2} \mathrm{PO}_{4}$ ”, J. Appl. Phys. 73, 7780 (1993).

3. K. Kobayashi, S. Endo, K. Deguchi, O. Shimomura, and T. Kikegawa, "Crystal systems and dielectric constants of high-pressure phases of $\mathrm{KH}_{2} \mathrm{PO}_{4}$," Phys. Rev. B 55, 2850 (1997). 